\title{
Magnetization Reversal Behaviour of Planar Nanowire Arrays of Fe
}

\author{
S.K. Arora ${ }^{1 *}$, B.J. O’Dowd ${ }^{1}$, P. Thakur ${ }^{2}$, N.B. Brookes ${ }^{2}$, B. Ballesteros ${ }^{3}$, P. Gambardella ${ }^{3,4}$ and I.V. Shvets ${ }^{1}$ \\ ${ }^{1}$ Centre for Research on Adaptive Nanostructures and Nanodevices (CRANN) and School of Physics, Trinity College Dublin, Dublin \\ 2, Ireland; ${ }^{2}$ European Synchrotron Radiation Facility, BP220, 38043 Grenoble Cedex, France; ${ }^{3}$ Catalan Institute of Nanotechnology \\ (ICN), UAB Campus, E-08193 Barcelona, Spain; ${ }^{4}$ ICREA and UniversitatAutonoma de Barcelona, E-08193 Barcelona, Spain
}

\begin{abstract}
Systematic investigations of magnetization behavior performed on planar arrays of 30 nm wide Fe-nanowires (NW) fabricated on oxidized step-bunched Si (111) templates reveal that the NW arrays exhibit an uniaxial anisotropy dominated by the shape of the wires, that facilitates in retaining the magnetization in-plane with easy axis parallel to the wires. The NWs possess polycrystalline character with bcc-crystal structure, and present an oxidized interface when capped with $\mathrm{MgO}$. From the temperature dependent magnetization studies we find that for thick wires $(>4.5 \mathrm{~nm})$ the magnetization reversal is governed by the curling reversal mode. Whereas, for thin wires the reversal is dominated by thermal activation.
\end{abstract}

Keywords: Magnetic nanowires, magnetization reversal, magnetic anisotropy, planar nanowire array, Self-Assembly, Vicinal surfaces.

\section{INTRODUCTION}

One dimensional (1D) magnetic structures such as nanowires, nanostripes, and atomic chains are of great importance due to their potential applications in the area of spintronics and as a test ground to investigate 1D magnetism [1-3]. The formation of such nanostructures can be either lithography based processes (top-down approach) or self-assembly based (bottom up approach). Though the former method is more definitive and controlled, the latter approach offers a greater throughput and possibilities of synthesizing structures well below the boundaries of current lithography capabilities $(\sim 20 \mathrm{~nm})$. Template mediated synthesis of magnetic nanowire (NW) arrays is quite popular. Variety of templates have been used to produce magnetic NW arrays of wire width ranging from single atom to several hundred nm [4-13]. Commonly used approaches to fabricate the periodic planar arrays of magnetic NWs are step flow, step decoration [7-11], and reactive deposition epitaxy (RDE) $[12,13]$ on vicinal templates. Major challenges related to forming planar NW arrays on self-assembled templates are the super-paramagnetism (due to small thickness of the NWs), and the material selective nature of the process, making it suitable only for certain NW material and substrate combinations. These limitations restrict their application potential. In self-assembled magnetic nanostructures, to overcome super-paramagnetism (SP), one needs to increase the strength of energy barrier opposing spontaneous magnetization flipping. It scales with $K V$, where $K$ is the magnetic anisotropy per unit volume, and $V$ the system's volume. This suggests that a possible route to overcome SP in the planar NW arrays is to either enhance $K$ (e.g., by deposition on a heavy metal substrate [14]) or enhance $V$ (by fabricating thick NW arrays [15-16]).

The latter approach has been successfully employed to produce room temperature (RT) ferromagnetic NW arrays using shallow angle deposition [15-16] and reactive deposition epitaxy techniques [12]. Considering the fundamental and applied interest in the magnetic nanostructrues, it is important to investigate the magnetization reversal processes in them. Moreover, the strong dependence of the reversal processes on the geometry (width, length, thickness, interwire separation etc.), associated length scales, and material characteristics calls for further studies in this area [1-3]. Only a few reports have focused on magnetization reversal in planar NW arrays

\footnotetext{
*Address correspondence to this author at the CRANN and School of Physics, Trinity College Dublin, Dublin-2, Republic of Ireland; Tel: +353-18963031; Fax: +353-1-8963037; E-mail: aroras@tcd.ie
}

defined lithographically [17]. Here, we report a systematic study of magnetization behavior of Fe-NW arrays of $30 \mathrm{~nm}$ wire width on oxidized vicinal Si (111) templates. The magnetic anisotropy of these NW arrays is found to be dominated by the shape of the NWs that keeps the magnetic easy axis in-plane and along the wire length. The magnetization reversal process in these planar NW arrays is found to be thickness dependent.

\section{EXPERIMENTAL}

Step-bunched vicinal Si templates used in the present study were prepared by performing a dc-current annealing [18]under ultrahigh vacuum (UHV) conditions of n-type doped vicinal Si (111) stripes with resistivity $1-10 \Omega \cdot \mathrm{cm}$. The Si substrates had a miscut of 4 degrees along the $(11 \overline{2})$ crystallographic direction. Annealing at $920^{\circ} \mathrm{C}$ (cooling rate of $0.02^{\circ} \mathrm{C} /$ second) with a direct current applied perpendicular to the step edges in the ascending step direction led to the formation of regular step bunches of $140 \mathrm{~nm}$ periodicity $(30 \mathrm{~nm}$ wide step-bunch $+110 \mathrm{~nm}$ wide terraces). The Si templates were oxidized (about $120 \mathrm{~nm}$ oxide thickness) using a standard thermal oxidation procedure carried out at $830^{\circ} \mathrm{C}$ for a duration of $15 \mathrm{hrs}$. A temperature lower than the $(1 \times 1)$ to $(7 \times 7)$ reconstruction cross-over temperature was selected to preserve the step-bunches.

The growth of $\mathrm{Fe}$-NWs was carried out at RT by depositing Fe $(0.02-0.04 \AA / \mathrm{s})$ onto the oxidized templates at a small angle $\left(0.5-4^{\circ}\right)$ using a multi-pocket e-beam evaporator (Telemark, USA) in a UHV chamber with a base pressure of better than $5 \times 10^{-10}$ Torr. The deposition flux was directed towards the uphill (ascending step) direction at a deposition angle of $3^{\circ}$ leading to formation of nanostripes covering the whole width of the step-bunched facet. This method of producing planar NW arrays on step-bunched templates was termed ATLAS (atomic terrace low angle shadowing) and its details are given elsewhere [16]. Two sets of Fe NW array samples used in the present study were prepared on step-bunched vicinal Si templates; for topographical investigations we prepared Fe-NW arrays without cap layer, whereas for magnetization and transmission electron microscopy (TEM) studies, the arrays were protected with a $5 \mathrm{~nm} \mathrm{MgO}$ cap layer. The templates produced under identical conditions were used for preparing the two sets of NW arrays. The average step-terrace periodicity obtained on these templates was reproducible to a large extent (up to $80 \%$ ), as determined from the statistics obtained from large area atomic force microscopy scans taken at several locations of the template surfaces. 
The surface topographic studies on the templates and uncapped Fe-NW arrays were performed using an atomic force microscope, (AFM, Solver Pro, NT MDT) and scanning electron microscope (SEM, Zeiss Ultra). For structural and interface studies a high resolution transmission electron microscope (HRTEM, FEI Tecnai F30 operated at $300 \mathrm{kV}$ ) was used. Sample cross-sections for HRTEM observation were prepared using focused ion beam (FIB) on a Helios Nanolab microscope. To examine the magnetic properties we used a vibrating sample magnetometer (Quantum Design- Physical Property Measurements System) with a sensitivity of $5 \times 10^{-7}$ emu. The diamagnetic contribution from the substrate was removed from the measured data by subtracting magnetization data of a substrate of similar dimensions. Element specific $\mathrm{x}$-ray absorption (XAS) and $\mathrm{x}$ ray magnetic circular dichroism (XMCD) experiments were carried out in total electron yield (TEY) mode at the ESRF's ID08 beamline.

\section{RESULTS AND DISCUSSIONS}

Prior to discussing the magnetization properties we present morphological and structural characterization results obtained on the Fe-NW arrays. Representative AFM images of $140 \mathrm{~nm}$ periodicity templates and a $3 \mathrm{~nm}$ thick Fe- NW array grown at RT with a deposition angle of 3 degrees on oxidized step-bunched Si (111) template of $140 \mathrm{~nm}$ periodicity are shown in Fig. (1). One notices that the templates are highly periodic and the $F e N W s$ formed on these step-bunched templates are quite regular with wires remaining straight up to $1 \mu \mathrm{m}$ in length for majority of the NWs, leading to a large length/width (aspect) ratio. The NW thickness was determined from the analysis of the height profiles of AFM images along the miscut direction (Fig.1c). Considering the self-assembly nature of the process, we observed also small statistical variations in step height and occasional presence of crossing and merging steps that can induce variations in height and width of the wires. In a previous communication, we demonstrated that using the ATLAS method one can tailor the wire width using appropriate deposition angle and template periodicity [16]. A cross-sectional TEM image of a $\mathrm{Fe}$ NW array $(5 \mathrm{~nm}$ thick, on a template with $110 \mathrm{~nm}$ average periodicity) grown under similar deposition condition but with deposition flux directed towards the descending step (downhill) direction is shown in Fig. (2). The width of the NWs varies between $90-120 \mathrm{~nm}$ in this particular sample, which is related to the statistical fluctuations in the periodicity of the self-assembled template. From the TEM analysis we infer that the $\mathrm{Fe}$-NWs possess polycrystalline nature and its crystal structure is $b c c$ (Figs. 2b-d). In some areas of $\mathrm{Fe}$-NWs in the vicinity of $\mathrm{MgO}$ cap layer and oxidized $\mathrm{Si}$ substrate (Figs. 2c and e), we find that the crystal structure is $f c c$ and resembles the $\mathrm{FeO}$ structure. Our observation is in line with the crystal structure and symmetry reported for the interfaces $\mathrm{Fe}$ form with oxides [19]. It is also known that the epitaxial strain induced by the interfaces could stabilize the metastable fcc-Fe phase [20] which remains stable up to a few monolayers of Fe (typically 3-6 ML of $\mathrm{Fe})$. However, we could not identify any regions with fcc-Fe structure in the Fe-NW arrays used in the present study.
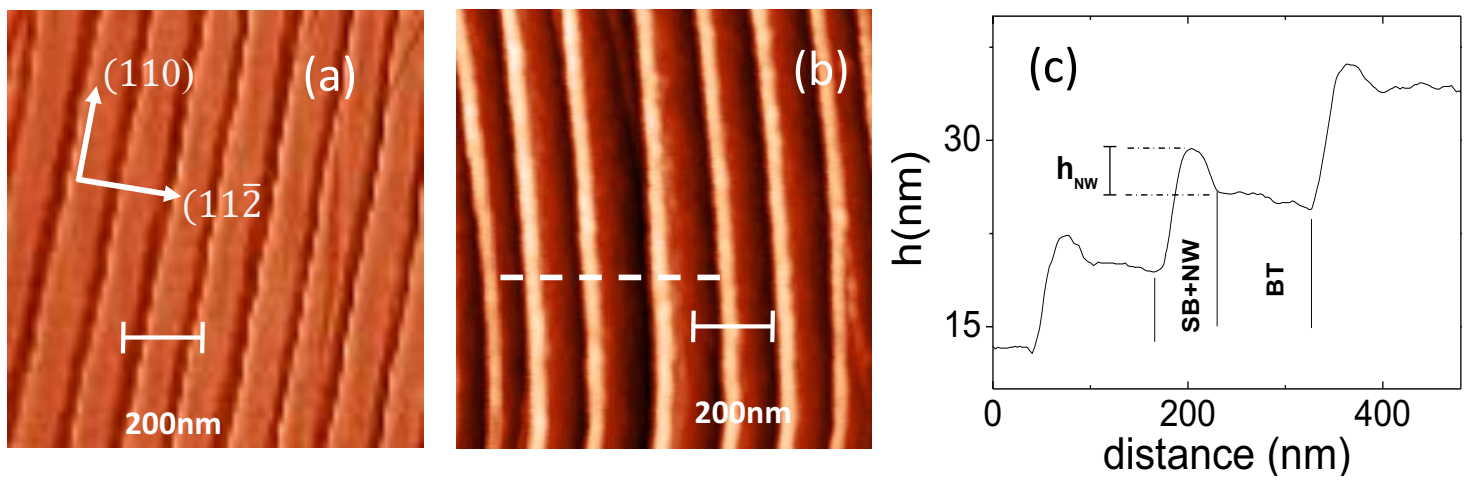

Fig. (1). (a) AFM phase image of a step-bunched Si (111) template with step-terrace periodicity of $140 \mathrm{~nm}$. (b) AFM height image of a Fe NW arrays grown using the ATLAS method at $300 \mathrm{~K}$ on oxidized step-bunched silicon templates with an average periodicity of $140 \mathrm{~nm}$. The Fe-flux is directed towards the ascending step direction (left to right direction in the images) at a deposition angle of $3^{\circ}$. (c) A height profile corresponding to the dashed line marked on the Fig. (1b) is shown in Fig. (1c). Labels BT, NW, SB and $\mathrm{h}_{\mathrm{NW}}$ Fig. (1c) denote bare terrace, nanowire, step bunch and NW height respectively.
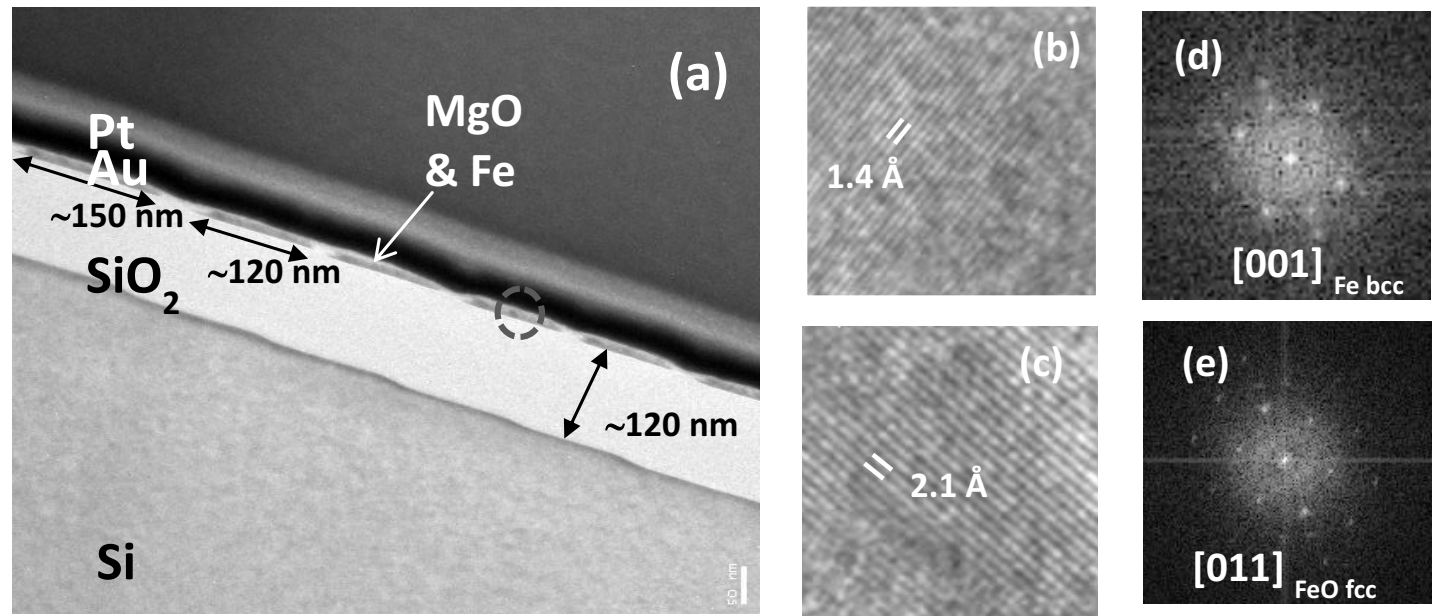

Fig. (2). (a) Cross-sectional TEM image of a 5nm thick Fe-NW array on a $110 \mathrm{~nm}$ average periodicity oxidized Si template. (b) High-resolution image showing the lattice of a Fe-NW crystallite with bcc structure. (c) A region of the Fe-NWs in the vicinity of SiO2 possessing fcc-structure which fits well with fccstructure of Fe-O (Wustite). (d) Fast Fourier Transform (FFT) of the region shown in image (b) showing the Fe bcc-crystal structure projected on its [001] zone axis. (e) Fast Fourier Transform (FFT) of the region shown in image (d) showing the fcc-crystal structure of Fe-O projected on its [011] zone axis. 


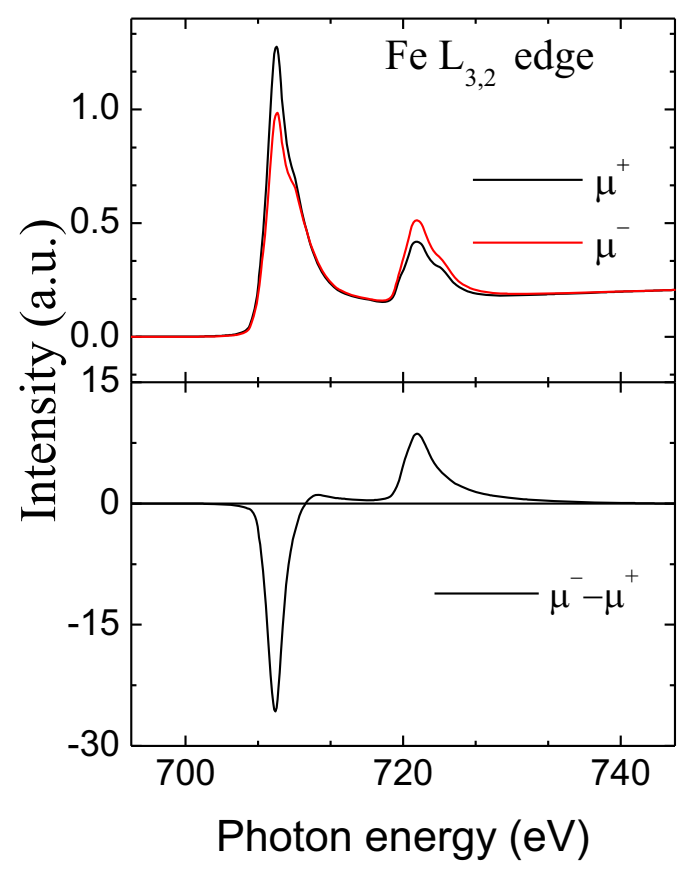

Fig. (3). (Color online) X-ray absorption spectra taken at the $\mathrm{Fe} L_{3,2}$ edge for an array of NWs with average width $40 \mathrm{~nm}$ grown on an oxidized silicon template with $110 \mathrm{~nm}$ periodicity. The spectra were recorded at $300 \mathrm{~K}$ with $\mathrm{X}$-rays incident parallel to the surface normal for magnetization parallel and antiparallel to the $\mathrm{x}$-ray helicity (black and red curves). The corresponding $\operatorname{XMCD}\left(\mu^{+}-\mu^{-}\right)$signal is shown in the lower panel.

Fig. (3) shows the X-ray absorption spectra of a $F e$-NW array (2.4 nm thick, $40 \mathrm{~nm}$ wide on $110 \mathrm{~nm}$ periodic template) after removal of the $\mathrm{MgO}$ cap layer using $\mathrm{Ar}$ ion etching. The spectra are taken at the $L_{2,3}$ edges of $\mathrm{Fe}$ in the TEY mode using negative and positive circularly polarized light with $99 \pm 1 \%$ polarization. A magnetic field (B) of $5 \mathrm{~T}$ was applied parallel to the $\mathrm{x}$-ray polarization direction to fully saturate the sample magnetization (M) along the $\mathrm{x}$-ray beam direction. The XAS spectra were normalized to the incident photon flux. XMCD spectra were obtained by switching either the x-ray polarization or M. Here, $\mu^{+}\left(\mu^{-}\right)$refers to the absorption coefficient for the photon helicity parallel (antiparallel) to the $\mathrm{Fe} 3 d$ majority spin direction. The XAS line shape is typical of $\mathrm{Fe}$ metal and shows signs of oxidation (as seen in the shoulder at 1.1 $\mathrm{eV}$ above the $\mathrm{Fe} L_{3}$ edge peak at $708.36 \mathrm{eV}$ ) owing to the presence of an oxidized interface with the cap layer of $\mathrm{MgO}$, in agreement with the TEM data. The magnitude of the XMCD signal, $\left(\mu^{-}-\mu^{+}\right)$, compared to the average XAS, $\left(\mu^{-}+\mu^{+}\right) / 2$, confirms the presence of a sizeable magnetic moment. We perform sum rule analysis of the observed XAS spectra to extract the spin and orbital magnetic moments per $\mathrm{Fe}$ atom [21, 22]. We estimate that the average spin magnetic moment $\left(m_{s}\right)$ of the Fe nanowires amount to $0.98(1.04)$ $\mu_{\mathrm{B}} /$ atom at $300 \mathrm{~K}(10 \mathrm{~K})$. Whereas value of the orbital magnetic moment $\left(m_{\nu}\right)$ is $0.052(0.071) \mu_{\mathrm{B}} /$ atom at $300 \mathrm{~K}(10 \mathrm{~K})$.In this analysis we assume a negligible contribution of spin dipole moment $\left(m_{T}\right)$ based on the fact that its magnitude is quenched due to crystal field and hybridization effects in $\mathrm{Fe}$ [21]. Much reduced values of effective spin moment as compared to those reported for bulk bcc$\mathrm{Fe}$ are related to the presence of oxidized interface of $\mathrm{Fe}$ with cap layer $(\mathrm{MgO})$ and substrate $\left(\mathrm{SiO}_{2}\right)$ [23]. Note that due to the relatively large thickness of the wires, dimensionality effects that are known to enhance $m_{s}$ and $m_{l}$ in ultrathin atomic chains [8] do not play a major role here.

To understand the role of various anisotropies influencing the magnetic properties of the NW arrays, we performed temperature dependent $(300 \mathrm{~K}-10 \mathrm{~K})$ magnetization studies on Fe- NW arrays with $30 \mathrm{~nm}$ average width, $140 \mathrm{~nm}$ periodicity, and capped with $5 \mathrm{~nm} \mathrm{MgO}$. The Fe wires have thickness of 3, 4.5 and $6.1 \mathrm{~nm}$ and 9 $\mathrm{nm}$, hereafter referred to as Sample 1, 2, 3, and 4, respectively. For these NW arrays used for magnetization studies we select an interwire separation $(s)$ to wire width $(w)$ ratio greater than three. The choice of this $s / w$ ratio is linked to the fact that for this ratio, effect of the inter-wire magnetostatic interactions would be negligible and NW within the arrays could be considered as non-interacting [24]. The magnetization hysteresis (M-H) loops of all the Fe-NW arrays measured at $300 \mathrm{~K}$ with an in-plane applied field directed either along $\left(\mathrm{H}_{\|}\right)$or across $\left(\mathrm{H}_{\perp}\right)$ the wires (step-edges) are shown in Fig. (4). The values of $\mathrm{H}_{\mathrm{C} \|}\left(\mathrm{H}_{\mathrm{C} \perp}\right)$ are found to be 90(55), 260 (80), 977(147), and 700(180) Oe for Samples 1, 2, 3, and 4 respectively. For all the samples an easier approach towards magnetic saturation is noticed for $\mathrm{H}_{\|}$as compared to $\mathrm{H}_{1}$, suggesting that the magnetic easy axis is along the wire length. The out of plane M-H loops (not shown) measured at $300 \mathrm{~K}$ were found to exhibit a hard axis behavior ruling out the presence of spin-reorientation transition. From the analysis of magnetization data, we infer that the value of coercivity, $\mathrm{H}_{\mathrm{C}}$, is thickness dependent. $\mathrm{H}_{\mathrm{C}}$ is found to increase with the wire thickness up to $6.1 \mathrm{~nm}$ (Sample 1-3), whereas for Sample 4 we notice a reduction in $\mathrm{H}_{\mathrm{C}}$. Another point to notice is that $\mathrm{H}_{\mathrm{C}}$ of all the samples is enhanced compared to $\mathrm{H}_{\mathrm{C}}\left(\mathrm{H}_{\mathrm{C}}\right.$ values was found to be 20 and 40 Oe for 3 and $5 \mathrm{~nm}$ thick Fe films respectively) of the Fe film of similar thickness grown on a flat $\mathrm{Si}$ (111) substrate under normal deposition conditions. The observation of a reduced $\mathrm{H}_{\mathrm{C}}$ for Sample 4 is in line with the general expectation of enhanced magnetostatic interactions among the wires due to increase in the strength of dipolar fields with increasing thickness. We would like to point out that the $\mathrm{MgO}$ cap layer is required to prevent oxidation and degradation of the Fe-NW surfaces from exposure to air. The $\mathrm{MgO}$ cap layers used to protect the $\mathrm{Fe}-\mathrm{NWs}$ are found to form an interface oxide layer $(\sim 1 \mathrm{~nm})$ with the Fe-NWs (as evident from the XAS sepectra (Fig. 3)), taken this into account there was no noticeable change in magnetization of the Fe-NW array. Moreover, the Fe-MgO interface is known to be chemically stable with a few monolayers of the $\mathrm{Fe}$ transformed into $\mathrm{Fe}-\mathrm{O}$ [25]. However, long term aging in our case reduces the magnetization of the NWs by $10-15 \%$ in about 8 12 week after deposition.

From the temperature dependent $(300-10 \mathrm{~K})$ magnetization studies we find that the magnitude of $\mathrm{H}_{\mathrm{C}}$ and $\mathrm{M}_{\mathrm{R}}$ for all the samples increases with the decreasing temperature (results are summarized in Table I). The increase in magnitude of $\mathrm{H}_{\mathrm{C}}$ is more noticeable for the thinner sample (for Sample 1, its magnitude changes from 90 Oe to 628 Oe when cooled from $300 \mathrm{~K}$ to $10 \mathrm{~K}$ ). This points to important role of thermal fluctuations in determining the magnetization behavior of these NW arrays. Relatively much smaller enhancement in $\mathrm{H}_{\mathrm{C}}$ was observed for the thicker sample (for Sample $3, \mathrm{H}_{\mathrm{C} \|}$ increases from $977 \mathrm{Oe}$ to $1380 \mathrm{Oe}$ ) when cooled from $300 \mathrm{~K}$ to $10 \mathrm{~K}$. For all the samples only a marginal increase $(\sim 5-10 \%)$ in the magnitude of the saturation magnetization $\left(\mathrm{M}_{\mathrm{s}}\right)$ was observed with a decrease in temperature from $300 \mathrm{~K}$ to $10 \mathrm{~K}$, suggesting that the Curie temperature of the NWs is well-above $300 \mathrm{~K}$. Observed changes in the $M_{s}$ are consistent with the modifications observed with XMCD measurements. From the magnetization results of the NW arrays, we infer that the shape anisotropy contribution to the effective anisotropy is the dominant one. Due to the polycrystalline nature of the nanowires, contribution from the magnetocrystalline and magneto-elastic anisotropies to the effective magnetic anisotropy is expected to be negligible. This shape related uniaxial anisotropy is preserved at low temperatures (down to $10 \mathrm{~K}$ ). We also find that for all samples the $\mathrm{H}_{\mathrm{C} \mid}>\mathrm{H}_{\mathrm{C} \perp}$, which is in agreement with the shape anisotropy origin of the enhanced $\mathrm{H}_{\mathrm{C}}$ as discussed qualitatively within the Stoner-Wohlfarth model [6].

For large aspect ratio wires (as is our case) one would expect that the hysteresis loop (HL) along the wire would be perfectly square with $100 \%$ remnance $\left(\mathrm{M}_{\mathrm{R}}\right)$. In our case the $\mathrm{HL}$ are not square 

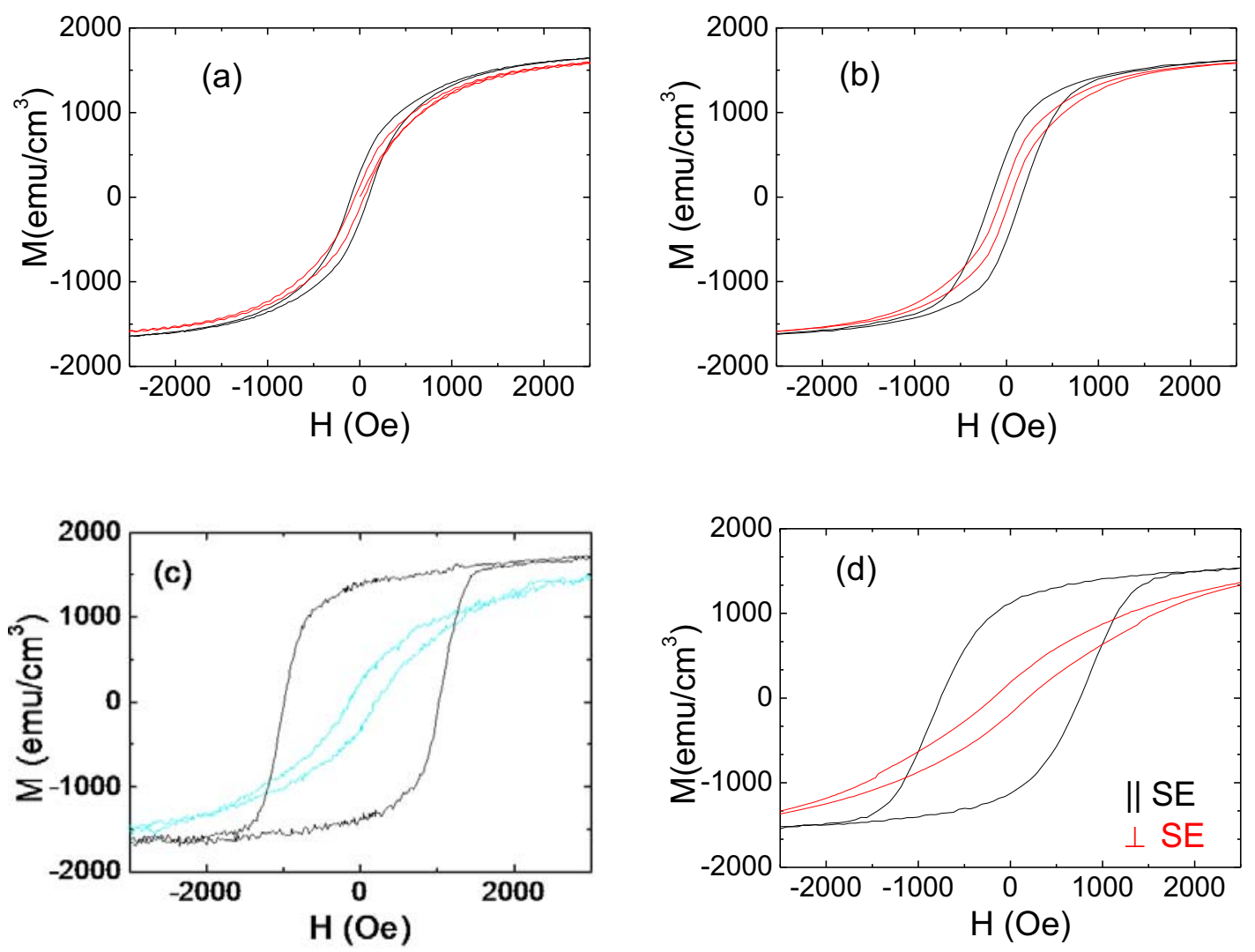

Fig. (4). (Color online) Magnetization hysteresis loops measured at $300 \mathrm{~K}$ with an in-plane magnetic field directed either along $\left(\mathrm{H}_{\|}\right)$or across $\left(\mathrm{H}_{\perp}\right)$ the length of the NWs for (a) Sample 1, (b) Sample 2, (c) Sample 3, and (d) Sample 4.

Table I. NW Thickness (t), Coercivity $\left(H_{C}\right)$, and Remnant Magnetization $\left(M_{R}\right)$ Values Measured at 300 and $10 \mathrm{~K}$ for All the Samples with the Magnetic Field Applied in-Plane Along $\left(\mathrm{H}_{\|}\right)$and $\operatorname{Across}\left(\mathrm{H}_{\perp}\right)$ the NWs

\begin{tabular}{|c|c|c|c|c|c|c|c|c|c|}
\hline \multirow{3}{*}{ Sample } & \multirow{3}{*}{$\mathrm{t}(\mathrm{nm})$} & \multicolumn{4}{|c|}{ Hc (Oe) } & \multicolumn{4}{|c|}{ MR (\%) } \\
\hline & & \multicolumn{2}{|c|}{$300 \mathrm{~K}$} & \multicolumn{2}{|c|}{$10 \mathrm{~K}$} & \multicolumn{2}{|c|}{$300 \mathrm{~K}$} & \multicolumn{2}{|c|}{$10 \mathrm{~K}$} \\
\hline & & $\mathbf{H}_{\|}$ & $\mathbf{H}_{\perp}$ & $\mathbf{H}_{\|}$ & $\mathbf{H}_{\perp}$ & $\mathbf{H}_{\|}$ & $\mathbf{H}_{\perp}$ & $\mathbf{H}_{\|}$ & $\mathbf{H}_{\perp}$ \\
\hline Sample 1 & 3 & 90 & 55 & 628 & 252 & 17 & 7.5 & 43 & 28 \\
\hline Sample 2 & 4.5 & 260 & 80 & 614 & 300 & 30 & 9.2 & 46.5 & 27 \\
\hline Sample 3 & 6.1 & 977 & 147 & 1380 & 360 & 81 & 9.5 & 79.6 & 26 \\
\hline Sample 4 & 9.0 & 700 & 180 & 1012 & 290 & 59 & 11 & 67 & 20 \\
\hline
\end{tabular}

and $M_{R}$ is also much smaller than $100 \%$. Further, $H_{C}$ is one order of magnitude smaller than the anisotropy field $\mathrm{H}_{\mathrm{A}}=2 \pi \mathrm{M}_{\mathrm{s}}=11 \mathrm{kOe}$ which suggests that one requires a deeper understanding of the magnetization reversal mechanism of the NW arrays. To understand the above results, we consider the sphere chain model [26] in which the NWs are considered to be formed by a linear chain of noninteracting single domain particles whose magnetization state is determined by the magnetostatic interaction between the spheres (exchange interactions are not taken into account). The estimated value of $\mathrm{H}_{\mathrm{C} \|}$ using this simple model is $2.5 \mathrm{kOe}$, which is still a factor of 2 greater than the observed $\mathrm{H}_{\mathrm{C} \|}$. According to this simple model, $\mathrm{H}_{\mathrm{C}}$ should follow the temperature dependence of $\mathrm{M}_{\mathrm{S}}$. However, this is not the case, as $M_{S}$ changes only marginally within the whole temperature range whereas $\mathrm{H}_{\mathrm{C}}$ exhibits much greater changes with temperature. These results suggest that the changes in $\mathrm{H}_{\mathrm{C}}$ could be attributed not only to the modification of $\mathrm{M}_{\mathrm{S}}$ with temperature, but also to thermal fluctuations. To investigate the effect of thermal activation on the magnetization reversal mechanism, we consider the case where the magnetic anisotropy is dominated by the shape-anisotropy contribution. In such a situation, the variation of $\mathrm{H}_{\mathrm{C}}$ with temperature can be writtenas [4, 27].

$$
H_{c}(T)=H_{c}(0) \frac{M_{S}(T)}{M_{S}(0)}\left\{1-\left\lfloor\frac{25 k_{B} T M_{S}^{2}(0)}{E_{o} M_{S}^{2}(T)}\right\rfloor^{\frac{1}{\alpha}}\right\}
$$

where $\mathrm{H}_{\mathrm{C}}(0)$ is the coercivity at $0 \mathrm{~K}, \mathrm{E}_{\mathrm{o}}$ is the energy barrier for reversal related to the shape anisotropy, $\mathrm{M}_{\mathrm{s}}(\mathrm{T})$ and $\mathrm{M}_{\mathrm{s}}(0)$ are the saturation magnetization at $\mathrm{T}$ and $0 \mathrm{~K}$, respectively. The value of the exponent $\alpha$ is related to the magnitude of anisotropy and distri- 


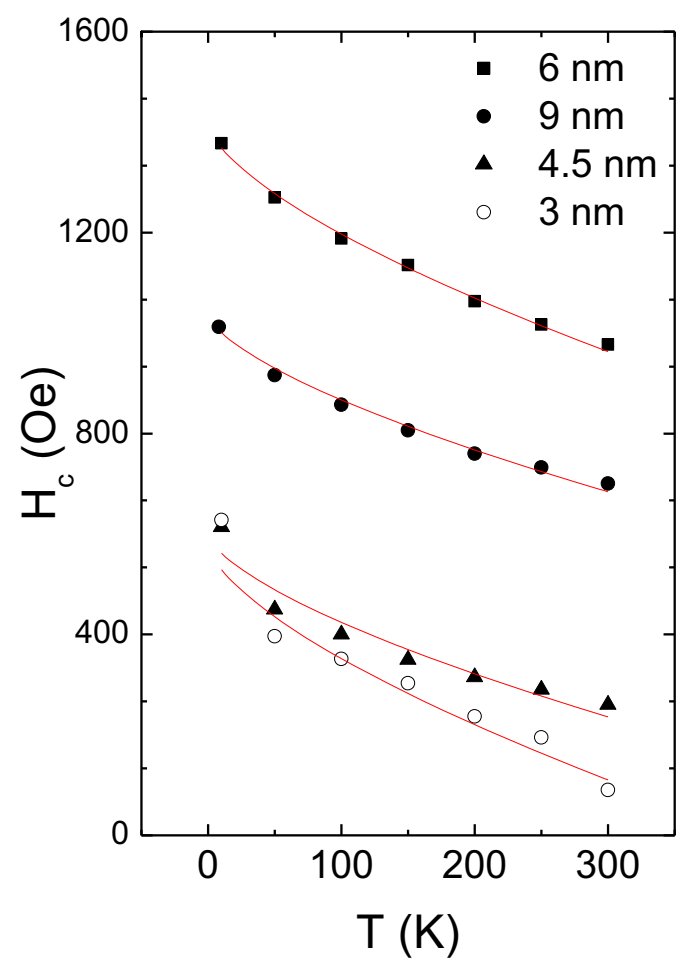

Fig. (5). (Color online) Variation of $\mathrm{H}_{\mathrm{C}}$ as a function of temperature measured with the in-plane field applied along $\left(\mathrm{H}_{\|}\right)$the wires for all the samples. Also shown in the figure are the fitted curves using equation 1 with $\alpha=$ 0.66 .

bution of the anisotropy axes relative to the applied field. Its value for the curling mode and coherent rotation reversal mode are $3 / 2$ and 2 respectively. We find that the magnitude of $\mathrm{H}_{\mathrm{C} \|}$ increases sharply for the thinner sample (Sample 1) as opposed to thicker sample (Sample 3), as seen in Fig. (5). We further fit the $\mathrm{H}_{\mathrm{C} \|}$ versus $\mathrm{T}$ data with equation 1 using $\alpha=3 / 2$. The choice of $\alpha=3 / 2$ which corresponds to the curling mode reversal and related to the fact that the wire widths for our samples are above the predicted critical width for coherent magnetization reversal in Fe [4] [ 12 nm $\left(d_{c}=3.68 \sqrt{ }\left(A / \pi M_{s}^{2}\right]\right)$.In our analysis, we neglect the variation in $\mathrm{M}_{\mathrm{S}}$ with $\mathrm{T}$, as it remains nearly constant within the temperature range studied $(5-12 \%$ increase with temperature decreasing to $10 \mathrm{~K})$. From fitting the experimental data with equation 1, we estimate the activation energies $\left(\mathrm{E}_{0}\right)$ to be 1.2 and $5.5 \mathrm{eV}$ for Sample1 and Sample 3 , respectively. From the values of $\mathrm{E}_{0}$ and $\mathrm{H}_{\mathrm{C} 0}$ obtained by the fits, we can determine the activation volume $\left(\mathrm{V}^{*}\right)$ involved in the magnetization reversal process using the relation $E_{0}=\mu_{0} H_{C 0} M_{S 0} V^{*}$. We find $\mathrm{V}^{*}=1780$ and $3580 \mathrm{~nm}^{3}$ for Sample 1 and Sample 3, respectively. Much smaller values of $\mathrm{V}^{*}$ compared to the NW's volume (please see Table II) indicate that the reversal is localized within $\mathrm{V}^{*}$ and the magnetization does not reverse coherently in the whole NW volume. The poly-crystalline nature of the wires and surface related structural imperfections could produce localization of the reversal modes [28]. From the above analysis, for thickber NW arrays we find a qualitative agreement with the model (eq.1), while the situation for thinner wires (Sample 1) is more complex. The disagreement between of model predictions for thinner samples could be understood from the fact that the small size nano-islands (average $\sim 5-10 \mathrm{~nm}$ ) that constitute the NWs are of size comparable to critical diameter for coherent reversal. This suggests that the individual islands switch their magnetization via coherent rotation. However, the presence of magnetostatic and exchange interactions among the crystal grains constituting the wires complicates the reversal leading a stronger deviation from the predictions of equation 1 .
Table II. Summary of the Parameters Derived from the Fitting of $\mathbf{H}_{C}$ Versus $T$ Dependence Using Equation 1

\begin{tabular}{|c|c|c|c|}
\hline Sample & $\mathbf{H}_{\mathbf{C}}(\mathbf{0})(\mathbf{O e})$ & $\mathbf{E}_{\mathbf{o}}(\mathbf{e V})$ & $\mathbf{V}^{*}\left(\mathbf{n m}^{\mathbf{3}}\right)$ \\
\hline \hline Sample 1 & 646 & 1.216 & 1780 \\
\hline Sample 2 & 600 & 2.12 & 3230 \\
\hline Sample 3 & 1417 & 5.56 & 3582 \\
\hline Sample 4 & 1038 & 5.05 & 4438 \\
\hline
\end{tabular}

\section{CONCLUSIONS}

In summary, we have shown through TEM and XAS studies that the planar NW arrays of $F e$ with $30 \mathrm{~nm}$ wire width and varying thickness fabricated using ATLAS method on self-assembled templates possess bcc-Fe structure and present oxidized interfaces with the substrate and cap layer. Bulk magnetometry and elementselective XMCD techniques reveal that these NW arrays are ferromagnetic at RT and exhibit an in-plane anisotropy with easy axis along the length of the wires. The magnetic properties of the FeNW arrays are dominated by their shape, which leads to an enhanced $\mathrm{H}_{\mathrm{C}}$ and longitudinal easy axis in the whole temperature range between $10 \mathrm{~K}$ and $300 \mathrm{~K}$. Our analysis suggest that the magnetization reverse with curling mode for thick nanowire $(t>4.5$ $\mathrm{nm}$ ), whereas for smaller thickness NWs reversal is influenced by the inherent morphology and surface imperfections, leading to a complex reversal behavior that is neither coherent nor curling.

\section{CONFLICT OF INTEREST}

The authors confirm that this article content has no conflicts of interest.

\section{ACKNOWLEDGEMENTS}

Work supported by the Science Foundation Ireland (SFI) under Contract No. 06/IN.1/I91, ERA-Net NANOWAVE program, the Irish Government's Program (ISPIRE) for Research in Third Level Institutions, Cycle 4, National Development Plan 2007-2013, Naughton Fellowship Program, the Spanish Ministerio de Ciencia y Innovación (EUI2008-03884 and PTA2008-1108-I), Agència de Gestió d'Ajuts Universitaris i de Recerca (2009 SGR 695), and Nanoaracat.

\section{DISCLOSURE}

Part of the information related to the model used for magnetization analysis in this article has been published in a study related to Co nanowire arrays in "S K Arora et al. 2012 Nanotechnology 23 235702 doi:10.1088/0957-4484/23/23/235702",

\section{REFERENCES}

[1] Schio, P.; Vidal, F.; Zheng, Y.; Milano, J.; Fonda, E.; Demaile, D.; Vodungbo, B.; Varalda, J.; de Oliveria, A.J.A.; Etgens, V.H. Magnetic response of cobalt nanowires with diameter below 5nm. Phys. Rev. B, 2010, 82, 094436.

[2] Carbone, C.; Gardonio, S.; Moras, P.;Lounis, S.; Heide, M.; Bihlmayer, G.; Atodiresei, N.; Dederichs, P. H.; Blügel, S.; Vlaic, S.; Lehnert, A.; Ouazi, S.; Rusponi, S.; Brune, H.; Honolka, J.;Enders, A.; Kern, K.; Stepanow, S.; Krull, C.; Balashov, T.; Mugarza, A.; Gambardella, P. Self-Assembled Nanometer-Scale Magnetic Networks on Surfaces: Fundamental Interactions and Functional Properties. Adv. Funct. Mater., 2011, 21, 1212-1228.

[3] Enders, A.; Skomski, R.; Honolka, J. Magnetic surface nanostructures. $J$. Phys. Condens. Matter., 2011, 22(43), 433001.

[4] Zheng, H.; Skomski, R.; Menon, L.; Liu, Y.; Bandyopadhyay, S.; Sellmyer, D. J. Structure and magnetic properties of ferromagnetic nanowires in selfassembled arrays. Phys. Rev. B, 2002, 65,134426.

[5] Thrun-Albrecht, T.; Schotter, J.;Kastle, G.; Emley, N.; Shibauchi, T.; KrusinElbaum, L.; Guarini, K.; Black, C.T.; Tuominen, M.T.; Russel, T.P. Ultra- 
high-Density Nanowire Arrays Grown in Self-Assembled Diblock Copolymer Templates. Science, 2000, 290(5499), 2126-2129.

[6] Sanchez-Barriga, J. ; Lucas, M. ; Radu, F. ; Martin, E. ; Multigner, M. Interplay between the magnetic anisotropy contributions of cobalt nanowires. Phys. Rev. B, 2009, 80(18), 184424

[7] Col, S.D.; Darques, M; Fruchart, O.; Cagnon, L. Reduction of magnetostatic interactions in self-organized arrays of nickel nanowires using atomic layer deposition. Appl. Phys. Lett., 2011, 98(11), 112501.

[8] Gambardella, P.; Dallmeyer, A.; Maiti, K.; Malagoli, M.C.; Eberhardt, W.; Kern, K.; Carbone, C. Ferromagnetism in one-dimensional monatomic metal chains. Nature, 2002, 416(6878), 301-304.

[9] RihunaCheng, K.; Guslienko, Yu; Fradin, F.Y.; Pearson, J.E.; Ding, H.F.; Li, Dongqi; Bader, S.D. Step-decorated Ferromagnetic Fe Nanostripes on Pt(997). Phys. Rev. B, 2005, 72(1), 014409.

[10] Elmers, H.J.; Hauschild, J.;Höche, H.; Gradmann, U.; Bethge, H.; Heuer, D.; Köhler, U. Submonolayer Magnetism of $\mathrm{Fe}(110)$ on W(110): Finite Width Scaling of Stripes and Percolation between Islands. Phys. Rev. Lett., 1994, 73(6), 898.

[11] Jubert, P.O.; Fruchart, O.; Meyer, C. Magnetic properties of step-decorated Fe nanostripes and dots grown on Mo(1 10$)$. J. Magn. Magn. Mater., 2002, 242, 565-567.

[12] Fruchart, O.; Eleoui, M.; Vogel, J.; Jubert, P.O.; Locatelli, A.; Ballestrazzi, A. Nanometers-thick self-organized Fe stripes: bridging the gap between surfaces and magnetic materials. Appl. Phys. Lett., 2004, 84(8), 1335-1337.

[13] Radican, K.; Berdunov, N.; Shvets, I.V. Studies of the periodic faceting of epitaxial molybdenum oxide grown on Mo(110). Phys. Rev. B, 2008, 77(8), 085417.

[14] Gambardella, P.; Dallmeyer, A.; Maiti, K.; Malagoli, M.C.; Rusponi, S.; Ohresser, P.; Eberhardt, W.; Carbone, C.; Kern, K. Oscillatory Magnetic Anisotropy in One-Dimensional Atomic Wires. Phys. Rev. Lett., 2004, 93(7), 077203.

[15] Oster, J.; Kallamayer, M.; Wiehl, L.; Elmers, H.J.; Adrian, H. Crystallography, morphology, and magnetic properties of Fe nanostructures on faceted $\alpha-\mathrm{Al}_{2} \mathrm{O}_{3} m$ plane. J. Appl. Phys., 2005, 97(1), 014303.

[16] Arora, S.K.; O’Dowd, B.J.; B Ballesteros, B.; Gambardella, P.; Shvets, I.V. Magnetic properties of planar nanowirearrays of Co fabricated on oxidizedstep-bunched silicon templates. Nanotechnology, 2012, 23(23), 235702.

[17] Goolaup, S.; Adeyeye, A.O.; Singh, N.; Gubbiotti, G. Magnetization switching in alternating width nanowire arrays. Phys. Rev. B, 2007, 75, 144430.

[18] Gibbons, B.J; Noffsinger, J; Pelz, P. Influence of Si deposition on the electromigration induced step bunching instability on $\operatorname{Si}\left(\begin{array}{lll}1 & 1 & 1\end{array}\right)$. Surf. Sci., 2005, 575(1-2), L51.

[19] Fan, Y.; Smith, K. J.; Lupke, G.; Hanbicki, A. T.; Goswami, R.; Li, C. H.; Zhao, H. B.; Jonker, B. T., Exchange bias of the interface spin system at the $\mathrm{Fe} / \mathrm{MgO}$ interface. Nat. Nanotechnol., 2013, 8(6), 438.

[20] Lin, M.T.; Shen, J.; Kuch, W.; Jenniches, H.; Klaua, M.; Schneider, C. M.; Kirschner, J. Structural transformation and spin-reorientation transition in epitaxial $\mathrm{Fe} / \mathrm{Cu}_{3} \mathrm{Au}(100)$ ultrathin films. Phys. Rev. B, 1997, 55(9), 5886

[21] Chen, C.T.; Idzerda, Y.U.; Lin, H.J.; Smith, N.V.; Meigs, G.; Chaban, E.; Ho, G.H.; Pellegrin, E.; Sette, F. Experimental Confirmation of the X-Ray Magnetic Circular Dichroism Sum Rules for Iron and Cobalt. Phys. Rev. Lett., 1995, 75, 152-155.

[22] Carra, P.; Thole, B.T.; Altarelli, M.; Wang. X. X-ray circular dichroism and local magnetic fields. Phys. Rev. Lett., 1993, 70(5), 694-697.

[23] Regan, T.J.; Ohldag, H.; Stamm, C.; Nolting, F.; Luning, J.; Stohr, J.; White, R.L. Chemical effects at metal/oxide interfaces studied by x-ray-absorption spectroscopy. Phys. Rev. B, 2001, 64, 214422.

[24] Adeyeye, A.O.; Bland, J.A.C.; Daboo, C.; Hasko, D.G. Magnetostatic interactions and magnetization reversal in ferromagnetic wires. Phys. Rev. B, 1997, 56(6), 3265-3270

[25] Oh, H.; Lee, S.B.; Seo, J.; Min, H.G.; Kim, J.S. Chemical Structure of the interface between $\mathrm{Fe}(001)$ and $\mathrm{MgO}$. Appl. Phys. Lett., 2003, 82(3), 361363 .

[26] Jacobs, I.S.; Beans, C.P. An Approach to Elongated Fine-Particle Magnets. Phys. Rev., 1955, 100(4), 1060-1067.

[27] He, L.; Chen, C. Effect of temperature-dependent shape anisotropy on coercivity for aligned Stoner-Wohlfarth soft ferromagnets. Phys. Rev. B, 2007, $75(18), 184424$.

[28] Skomski, R.; Zheng, H.; Zheng, M.; Sellmyer, D.J. Magnetic localization in transition-metal nanowires. Phys. Rev. B, 2000, 62(6), 3900-3904.
Received: December 4, $2012 \quad$ Revised: June 17, $2013 \quad$ Accepted: July 17, 2013 